\title{
Retroperitoneal myoma and chronic pelvic pain: case report and literature review
}

\author{
Malina Lukanova • Ehab Akkary • Yordan Popov • \\ Dimitar Stoykov • Margarita Nikolova
}

Received: 27 February 2009/Accepted: 25 March 2009/Published online: 21 April 2009

(C) Springer-Verlag 2009

\begin{abstract}
We present a case of a postmenopausal diabetic hypertensive female with a solitary retroperitoneal myoma $(2,025 \mathrm{~g})$ and no smooth muscle (myomatic) nodules on visceral and parietal peritoneum. She complained of chronic pelvic pain in absence of internal female genitalia (except uterine cervix), high frequency of urination, pelvic discomfort, and feeling of heaviness. The patient had undergone hysterectomy with bilateral adnexectomies 13 years ago and she had not had any exposure to exogenous hormones during her reproductive and postmenopausal period of life (oral contraception and hormonereplacement therapy). Several suppositions were made, supporting hormonal genesis of myoma with retroperitoneal
\end{abstract}

\author{
M. Lukanova $(\bowtie) \cdot$ Y. Popov \\ Department of Obstetrics and Gynaecology, \\ Medical University-Pleven, \\ 6 Byalo more str.-B-7, \\ 5800 Pleven, Bulgaria \\ e-mail: m_lukanova@yahoo.co.uk \\ E. Akkary \\ Minimally Invasive Surgery, Department of Surgery, \\ West Virginia University, \\ Morgantown, WV, USA \\ E. Akkary \\ Bariatric Surgery, Department of Surgery, \\ West Virginia University, \\ Morgantown, WV, USA \\ D. Stoykov \\ Visceral Surgery, Department of Surgery, \\ Medical University-Pleven, \\ Pleven, Bulgaria \\ M. Nikolova \\ Department of General and Clinical Pathology, \\ Medical University-Pleven, \\ Pleven, Bulgaria
}

localization in postmenopausal age and the significance of eventual presence of concomitant metabolic disturbance for its origin in that age was also emphasized. The known risk factors for growth and development of myoma were also affected by the presence of collateral dismetabolic conditions, which influenced and aggravated the disturbed hormonal balance. That is why they could be considered as a possible additional risk factor.

Keywords Retroperitoneal · Myoma $\cdot$ Leiomyosarcoma . Chronic pelvic pain

\section{Introduction}

Leiomyoma and leiomyosarcoma represent one of the various types of primary retroperitoneal masses in adults [1] with an overall incidence of $2.8 \%$; of those, the malignant variant, leiomyosarcoma, is $11.2 \%$ [2]. Retroperitoneal leiomyoma often presents a diagnostic challenge as it shares a resemblance with most retroperitoneal smooth muscle tumors in terms of clinical presentation, histology, and steroid/estrogen and progesterone/hormone positivity; moreover, they are considered to be malignant whereas retroperitoneal leiomyoma is not [3]. Diagnostic workup should include ultrasonography, CT, and/or MRI, but final diagnosis is obtained after a pathohistological examination has been performed.

\section{Case presentation}

We report a case of a 56-year-old diabetic hypertensive female, with body mass index (BMI) of $27.7 \mathrm{~kg} / \mathrm{m}^{2}$ and a waist-to-hip ratio of 2.4 , who presented to the clinic with 
increased urinary frequency, chronic pelvic pain, and discomfort of 6-7 months duration. The patient's past history was significant for three operative interventions in the following order: a cesarean section, a left-side adnexectomy with partial resection of the right ovary (endometriosis had been pathohistologically confirmed), and supravaginal hysterectomy with right-side cystadenexectomy secondary to uterine myoma and endometriotic cyst. Subsequently, she had amenorrhea for 13 years. The patient did not have a history of oral contraceptives (OC) use in the reproductive period of her life nor of hormonereplacement therapy (HRT) after the last operative intervention.

Gynecologic examination revealed a large irregular polylobular mass of hard consistency that engaged the pelvis and the lower part of the abdominal cavity to the level of the umbilicus. Upon inspection, the vaginal part of uterine cervix could not be visualized because the anterior vaginal wall was compressed by the mass. Laboratory blood work findings were within normal limits. Ultrasound examination revealed a solid $23.3 \times$ $17.4 \mathrm{~cm}$ non-homogenous pelvic mass with no invasion of surrounding structures. The patient underwent exploratory laparotomy. The mass was found to be originating from the pelvic floor and extending above the level of the umbilicus to the epigastrium and left upper quadrant. After extensive lysis of adhesions between the mass and the lateral and anterior abdominal walls, an approach to the retroperitoneal space was accomplished. The tumor was enucleated from the retroperitoneal space. A total absence of communication of the mass with the uterine cervix was found. There was no demonstrable connection to the genital tract and no tumors on the peritoneal surfaces were seen. Due to suspicion of sarcoma with retroperitoneal localization, an intraoperative histological examination was performed and the results indicated leiomyoma. The final gross pathological assessment was a tumor formation with overall dimensions of $23 \times 17 \times$ $11 \mathrm{~cm}$ and a weight of $2,050 \mathrm{~g}$. The tumor was whitishyellow, with a heavy lobulated surface and a hard elastic consistency (Fig. 1a, b). Histopahtological examination confirmed the diagnosis of retroperitoneal leiomyoma with edematous, myxoid, and inflammatory changes (Fig. 2a, b). Immunohistochemical staining of the tumor tissue was positive for estrogen and progesterone receptors (Fig. 3a, b).

\section{Discussion}

In 1952, leiomyomatosis peritonealis disseminata (LPD) was discovered byWilson and Peale; however, the first scientific description was attributed to H.D.Taubert in 1971
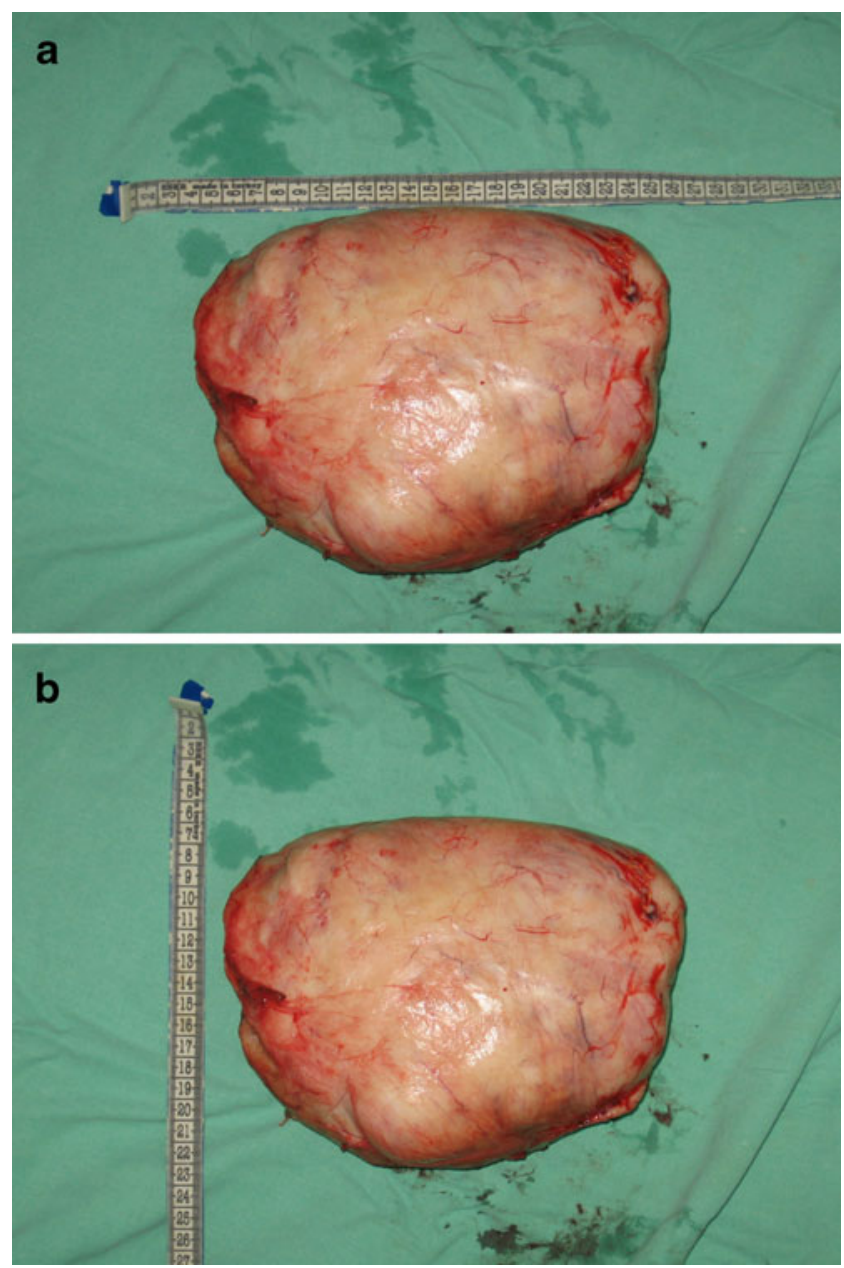

Fig. 1 a Macroscopic appearance of the retroperitoneal myoma (horizontally measured). b Macroscopic appearance of the retroperitoneal myoma (vertically measured)

[4]. LPD is pathological smooth muscle proliferation in the female pelvic peritoneum characterized by the presence of multiple tumors in peritoneal cavity that mimic a malignant process with metastases $[5,6]$. It is a rare condition found most commonly in women of reproductive age in association with pregnancy or OC use. Spontaneous regression is the usual clinical outcome following removal of the hormonal stimulus. Most cases are discovered incidentally during cesarean section procedures [6]. To the best of our knowledge, the case we report here is the first in the medical literature to describe a large retroperitoneal pelvic leiomyoma with no hormonal stimulus.

Dallenbach et al. hypothesized that the developing leiomyomatous nodules probably arise from the Müllerian epithelium, which is distributed throughout the subperitoneal mesenchyme. Individual predisposition and excessive hormonal stimulation permit the Müllerian derivatives to proliferate along lines of myofibrous differentiation. It is of clinical importance to realize that the dormant and pluripotent Müllerian epithelium in the 

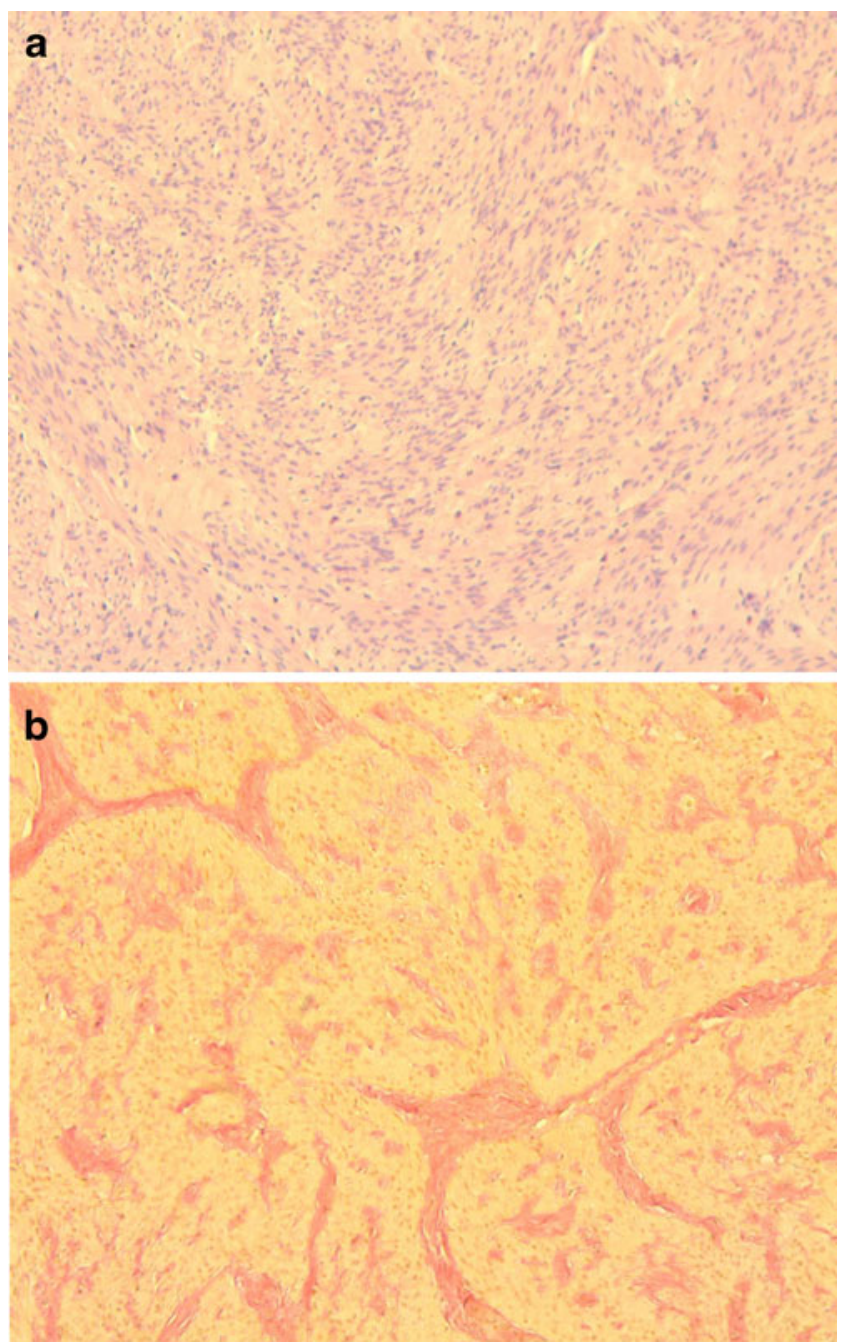

Fig. 2 a Retroperitoneal leiomyoma/H\&E. b Retroperitoneal leiomyoma/VG

minor pelvis may be stimulated by excessive hormones to proliferate $[7,8]$.

The hormonal factor was again re-emphasized by Danikas et al. considering LPD to be attributed to estrogen stimulation and seen only rarely in postmenopausal women. In such cases, pathogenesis is uncertain [9]. Stewart and Morton suggested a genetic basis for development of leiomyomata [10].

There are several suggested possible causes and concomitant conditions that might be responsible for the development of the peritoneal form of leiomyomatosis:

- Exposure to estrogens or increased endogenous estrogen levels $[5,11-13]$.

- Recurrent LPD after hysterectomy and bilateral salpingo-oophorectomy during combined HRT [14].

- In the presence of an ovarian Brenner tumor associated with tamoxifen use for the treatment of breast carcinoma $[9,15]$.
- LPD in the simultaneous presence of ovarian cystic teratoma [16].

- In association with estrogen-secreting ovarian fibrothecoma [17].

- Recurrent LPD exacerbated by in vitro fertilization [18].

- Long-term use of oral contraceptives [7, 13, 18].

- In association with endometriosis [13, 19].

- In constellation with other stigmas of Alpert's and Reed's syndrome [20, 21].

- Hereditary leiomyomatosis and renal cell cancer syndrome $[10,22]$.

- During pregnancy [7, 11, 18, 23].

- An unusual sensitivity of the coelomic tissues in patients with LPD in response to metaplastic stimuli [19].

Strinic et al. reported a case of a 65 -year-old nulliparous woman with normal BMI and no history of exogenous
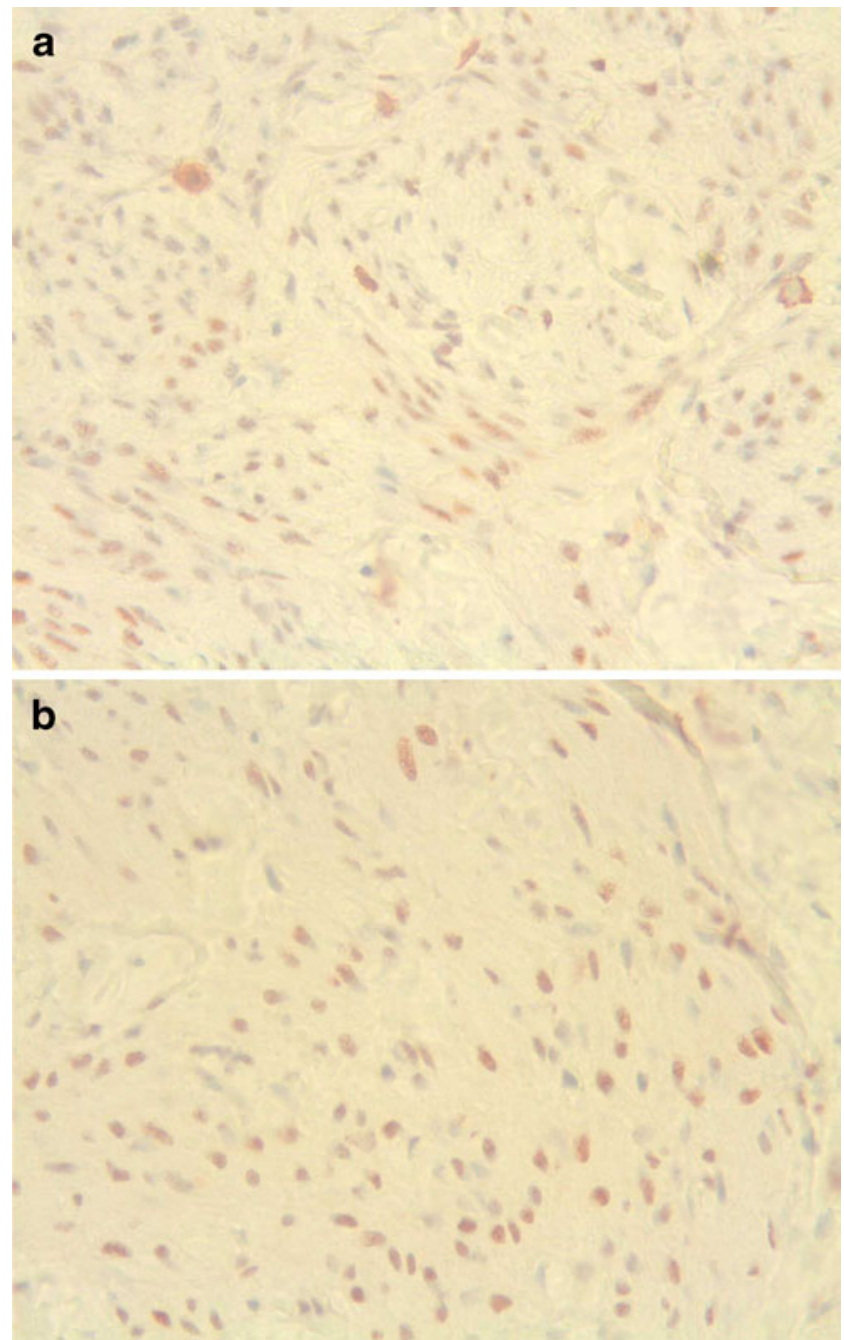

Fig. 3 a Estrogen and progesterone receptors of the retroperitoneal myoma/ER positivity. b Estrogen and progesterone receptors of the retroperitoneal myoma/PR positivity 
estrogens who presented with classical multiple LPD 16 years after menopause. The authors suggested that factors other than hormonal influences may contribute to pathogenesis of LPD [24]. Komatsu et al. described a case of LPD occurring in a postmenopausal woman who had undergone a total hysterectomy 15 years earlier and received no subsequent hormonal therapy. Intraoperatively innumerable tumor nodules ranging from 0.5 to $5.0 \mathrm{~cm}$ in diameter were found [25].

There is an interesting proposition for the origin of LPD in which LH receptors were present. Levels of FSH and (luteinizing hormone) LH increase after menopause and immunohistochemical analysis showed the presence of LH receptors, so the authors suggested that gonadotropins, rather than estrogen stimulation, might have contributed to development of LPD in that rare case [9].

The first description of a large retroperitoneal fibroleiomyoma was given by Zhitniuk et al. [26]. Abulafia and Sherer described a similar case of a large, asymptomatic retroperitoneal leiomyoma originating at the level of the pelvic floor and occupying the entire pelvis [27]. Iwaki et al. depicted a case of retroperitoneal myxoid leiomyoma in a 36-year-old woman; the tumor weighed $600 \mathrm{~g}$ and measured $16 \times 11 \times 9 \mathrm{~cm}$ [28].

Another case reported in the literature is a 76-year-old female who presented with history of pain on urination and urinary frequency. The patient was found to have a retroperitoneal tumor measuring $4 \times 4 \times 5.5 \mathrm{~cm}(35 \mathrm{~g})$. Twenty-seven years earlier, this patient had undergone surgical excision of a right retroperitoneal tumor that was histologically specified as a leiomyoma [29]. The last known case of retroperitoneal leiomyomatosis was presented by Dursun et al. in 2005 [30].

The case we report is of significant clinical interest due to several atypical presentations: the patient has been in amenorrhea for 13 years, she had undergone hysterectomy with bilateral adnexectomies without any exposure to exogenous hormones during her reproductive and postmenopausal periods, and the presence of a solitary myoma with no smooth muscle/myomatic/nodules on the visceral or parietal peritoneum.

In the depicted case the macroscopic characteristics of the formation resemble those of leiomyosarcoma. Leiomyomata are generally multiple, of variable size (3$5 \mathrm{~cm}$ ), firm, white, and infrequently associated with hemorrhage and necrosis (infarction type). Leiomyosarcoma is usually solitary, large, often $>10 \mathrm{~cm}$, soft, yellow or tan, and frequently associated with hemorrhage and necrosis (coagulative type) [31]. The mean age of presentation of leiomyosarcoma is about 10 years older than leiomyoma, with most women being older than 40 years [32].
We hypothesize that the development of the retroperitoneal myoma in this reported case can be based on the following theoretical formulations:

1. We make a supposition, based on the concept of Fredericks et al. of complex metaplastic phenomena affecting the female pelvis, that retroperitoneal myoma, like LPD, is one of the various epithelial and stromal lesions, which have been collectively termed 'müllerianosis'. According to the authors, the pelvic peritoneum in women maintains its ability to differentiate into specialized epithelia and stroma well into adult life, giving rise to the so-called secondary müllerian system, which differs from the primary müllerian system principally in its lack of organization. Another manifestation of this secondary system is the finding of smooth muscle aggregates in the subcoelomic stroma. Muscle (cells) derived from the secondary müllerian system typically indicate positivity for ER and PR whereas metaplastic leiomyocytes, not derived from the müllerian system, do not. The presence of estrogen receptors (ER) and progesterone receptors (PR) provide evidence for the müllerian origin of the muscle [6]. LPD is nodular proliferation of smooth muscle cells in the subcoelomic mesenchyme. Endometriosis is by far the most widely recognized 'lesion' of the secondary müllerian system. The associations found between endometriosis and leiomyomatosis suggest that these conditions may have a metaplastic origin from subperitoneal cells. Different hormonal environments may determine the direction which müllerian metaplasia takes [5].

The above facts suggest that, in the case described by us, the presence of prior endometriosis indicates the predisposition of the cells of subcoelomic mesenchyme to proliferation, particularly as the retroperitoneal myoma developed on a previously altered area, and confirms the hypersensitivity of the described anatomic structures to hormonal stimuli.

2. The impact of obesity as a risk factor for growth of myoma is due to its effect upon estrogen level and metabolism. The association between obesity and an increased incidence of uterine leiomyomata is reported in the literature. The risk of fibroids is increased by approximately $21 \%$ for each $10 \mathrm{~kg}$ increase in body weight [33]; a $6 \%$ increase in risk was reported for each unit increase in BMI $[34,35]$. Sato et al. observed that women with occult obesity $(\mathrm{BMI}<24.0$ and percent body fat $>=30 \%$ ) or women with upper-body fat distribution ( $>0.80$ waist-to-hip ratio) were at significantly higher risk $[6,35]$. Increased amounts of adipose tissue are represented by a higher BMI and especially 
by increased waist-to-hip ratio, which is a more precise indicator for corporal fat distribution and obesity among the elderly [36]. Abdominal visceral adipocytes are more metabolically active than abdominal subcutaneous adipocytes as they have high lypolytic activity and release large amounts of free fatty acids; this leads to increased serum levels of total or bioavailable estradiol with increasing BMI. Obesity is also associated with increased insulin level that reduces the synthesis and concentration of serum sex-hormonebinding globulin (SHBG) which increases the levels of bioavailable estrogens $[37,38]$.

BMI generally shows a direct linear relationship with insulin and estrogen levels and there are inverse associations of BMI and plasma insulin with SHBG [36, 39]. As for the waist-hip ratio factor, a negative association has been observed with circulating SHBG regardless of menopausal status. Women with a predominance of upper body or truncal fat have lower SHBG levels independent of overall adiposity [40].

In postmenopausal women, estrogens are produced from the conversion of precursor androgens or other estrogens, mainly in the adipose tissue, and their production is not regulated by feedback mechanisms. As a consequence, after menopause, estrogen concentrations are directly related to the amount of adipose tissue [36, 38]. Estrogen has been traditionally proposed as the primary promoter of uterine leiomyoma growth. This supposition has been based in part upon the clinical observations that fibroids occur only after menarche, develop during the reproductive years, may enlarge during pregnancy, and frequently regress following menopause. One hypothesis states that increased levels of estrogen and progesterone result in an increased mitotic rate that may contribute to myoma formation by increasing the likelihood of somatic mutations [37].

3. In the presented case, we also hypothesized that the concomitant dismetabolic condition, diabetes mellitus type 2, also played a role in the advent of the retroperitoneal myoma. This was based on the fact that diabetes, in addition to the excessive adipose tissue, had an impact on hormonal, particularly estrogen, metabolism and levels. Several studies have also shown that postmenopausal women with impaired glucose tolerance and type 2 diabetes have higher estrogen levels than postmenopausal women with normal glucose tolerance [41].

Among postmenopausal women, endogenous bioavailable testosterone, estradiol, and DHEA were positively associated with insulin resistance, whereas SHBG was negatively associated with insulin resistance [41]. Type-II diabetes is usually associated with insulin resistance and increased pancreatic insulin secretion for long periods both before and after disease onset [36]. In postmenopausal women, endogenous estradiol and free testosterone have been positively associated with glucose intolerance and type-II diabetes. Increasing levels of bioavailable testosterone and estradiol and decreasing levels of SHBG were associated with significantly increased odds of insulin-like growing factor and diabetes [41].

We hypothesize that the relative hyperestrogenism developed on the basis of metabolic disturbances and served as a promoter/initiator for growth and development of the retroperitoneal myoma on terrain with proven hypersensitivity (prior endometriosis) to hormonal stimuli.

\section{Conclusion}

Retroperitoneal leiomyomata are rare occurrences with few cases reported in the medical literature. Both the presented case and the available literature supported the hormonal genesis of myomata with retroperitoneal localization in the postmenopausal period including an emphasis on agerelated metabolic disturbance contributions to the origin site. The known risk factors for growth and development of myomata were also affected by the presence of concomitant dismetabolic conditions, which influenced and aggravated the disturbed hormonal balance.

Conflicts of interest We declare that the manuscript submitted to your journal, has not been published before and it is not under consideration for publication elsewhere. This manuscript has been approved by all co-authors. We confirm that all authors fulfilled all conditions required for authorship and approved the submission. The authors do not have a financial relationship with any organization or institution. We, the authors, have full control of all primary data.

\section{References}

1. Merran S, Karila-Cohen P, Vieillefond A (2004) Tumeurs rétropéritonéales primitives de l'adulte. J Radiol 85:252-264

2. Xu Y, Guo K, Guo R et al (2007) Surgical management of 143 patients with adult primary retroperitoneal tumor. World J Gastroenterol 13(18):2619-2621

3. Paal E, Miettinen M (2001) Retroperitoneal leiomyomas: a clinicopathologic and immunohistochemical study of 56 cases with a comparison to retroperitoneal leiomyosarcomas. Am J Surg Pathol 25(11):1355-1363

4. Taubert H, Wissner S, Haskins A (1965) Leiomyomatosis peritonealis disseminate: an unusual complication of genital leiomyomata. Obstet Gynecol 25:561-574

5. Sobiczewski P, Bidzinski M, Radziszewski J et al (2004) Disseminated peritoneal leiomyomatosis - case report and literature review. Ginekol Pol 75(3):215-220

6. Fredericks S, Russel P, Cooper M et al (2005) Smooth muscle in the female pelvic peritoneum: a clinicopathological analysis of 31 women. Pathology 37(1):14-21 
7. Dallenbach C, Miklaw H, Dallenbach F et al (1989) Disseminated peritoneal leiomyomatosis. A rare disease picture. Geburtshilfe Frauenheilkd 49(2):201-204

8. Klausch B, Wittstock G, Breuel I et al (1989) Disseminated peritoneal leiomyomatosis. A case report. Zentralbl Gynakol 111 (24):1624-1628

9. Danikas D, Vasilios T, Goudas C et al (2000) Luteinizing hormone receptor expression in leiomyomatosis peritonealis disseminate. Obstet Gynecol 95:1009-1011

10. Stewart E, Morton C (2006) The genetics of uterine leiomyomata. Obstet Gynecol 107(4):917-921

11. Bekkers R, Willemsen W, Schijf C et al (1999) Leiomyomatosis peritonealis disseminate: does malignant transformation occur? A literature review. Gynecol Oncol 75(1):158-163

12. Minassian S, Frangipane W, Polin J et al (1986) Leiomyomatosis peritonealis disseminata. A case report and literature review. J Reprod Med 31(10):997-1000

13. Herrero J, Kamali P, Kirschbaum M (1998) Leiomyomatosis peritonealis disseminata associated with endometriosis: a case report and literature review. Eur J Obstet Gynecol Reprod Biol 76 (2):189-191

14. Heinig J, Neff A, Cirkel U et al (2003) Recurrent leiomyomatosis peritonealis disseminata after hysterectomy and bilateral salpingooophorectomy during combined hormone replacement therapy. Eur J Obstet Gynecol Reprod Biol 111(2):216-218

15. Bristow R, Montz F (2001) Leiomyomatosis peritonealis disseminata and ovarian Brenner tumor associated with tamoxifen use. Int $\mathrm{J}$ Gynecol Cancer 11(4):312-315

16. Bourgain C, Pierre E, De Vits A et al (1994) Disseminated peritoneal leiomyomatosis. An unusual case. Pathol Res Pract 190 (5):500-504; discussion 504-506

17. Drake A, Dhundee J, Buckley C et al (2001) Disseminated leiomyomatosis peritonealis in association with oestrogen secreting ovarian fibrothecoma. BJOG 108(6):661-664

18. Deering S, Miller B, Kopelman J et al (2000) Recurrent leiomyomatosis peritonealis disseminata exacerbated by in vitro fertilization. Am J Obstet Gynecol 182(3):725-726

19. Kuo T, London S, Dinh T (1980) Endometriosis occurring in leiomyomatosis peritonealis disseminata: ultrastructural study and histogenetic consideration. Am J Surg Pathol 4(2):197-204

20. Mothes H, Heidet L, Arrondel C et al (2002) Alport syndrome associated with diffuse leiomyomatosis: COL4A5-COL4A6 deletion associated with a mild form of Alport nephropathy. Nephrol Dial Transplant 17(1):70-74

21. Renieri A, Bassi M, Galli L (1994) Deletion spanning the 5' ends of both the COL4A5 and COL4A6 genes in a patient with Alport's syndrome and leiomyomatosis. Hum Mutat 4(3):195-198

22. Kiuru M, Launonen V (2004) hereditary leiomyomatosis and renal cell cancer (HLRCC). Curr Mol Med 4(8):869-875

23. Fujii S, Okamura H, Nakashima N et al (1980) Leiomyomatosis peritonealis disseminata. Obstet Gynecol 55(3 Suppl):79S-83S

24. Strinič T, Kuzmič-Prusac I, Eterovič D et al (2000) Leiomyomatosis peritonealis disseminata in a postmenopausal woman. Arch Gynecol Obstet 264:97-98
25. Komatsu M, Tsuchiy S, Kuroda T et al (1996) Leiomyomatosis peritonealis disseminata occurring in a postmenopausal woman: report of a case. Surg Today 26(9):700-703

26. Zhitniuk R, Pomosov D, Seliuzhitskii I (1971) Large-sized retroperitoneal leiomyofibroma. Vestn Khir Im I I Grek 106 (5):127-128

27. Abulafia O, Sherer D (1995) Ultrasonographic and magnetic resonance imaging findings of a large asymptomatic retroperitoneal pelvic leiomyoma. Am J Obstet Gynecol 173(1):228-230

28. Iwaki H, Konishi $\mathrm{T}$, Okada $\mathrm{Y}$ et al (1995) Retroperitoneal myxoid leiomyoma: report of a case. Hinyokika Kiyo 41(10): 789-791

29. Kakizaki H, Yamaguchi T, Suzuki H et al (1989) A case report of retroperitoneal leiomyoma and review of the Japanese literature. Nippon Hinyokika Gakkai Zasshi 80(11): $1657-1660$

30. Dursun P, Salman M, Taskiran C (2005) Retroperitoneal leiomyomatosis: a case report. Int J Gynecol Cancer 15:1222-1225

31. Kurman R (2000) Mesenchymal tumors of the uterus. In: Kurman RJ (ed) Blauenstein's pathology of the female genital tract, 5th edn. Springer, New York, pp 561-617

32. Robboy S, Bentley R, Butnor K et al (2000) Pathology and pathophysiology of uterine smooth-muscle tumors. Environ Health Perspect 108(suppl 5):779-784

33. Ross R, Pike M, Vessey M et al (1986) Risk factors for uterine fibroids: reduced risk associated with oral contraceptives. Br Med J (Clin Res Ed) 293:359-362

34. Lumbiganon P, Rugpao S, Phandhu-fung S et al (1996) Protective effect of depot-medroxyprogesterone acetate on surgically treated uterine leiomyomas: a multicentre case-control study. Br J Obstet Gynaecol 103:909-914

35. Sato F, Nishi M, Kudo R et al (1998) Body fat distribution and uterine leiomyomas. J Epidemiol 8:176-180

36. Calle E, Kaaks R (2004) Overweight, obesity and cancer: epidemiological evidence and proposed mechanisms. Nat Rev Cancer 4(8):579-591

37. Flake G, Andersen J, Dixon D (2003) Etiology and pathogenesis of uterine leiomyomas: a review. Environ Health Perspect 111:1037-1054

38. Lukanova A, Lundin E, Zeleniuch-Jacquotte A et al (2004) Body mass index, circulating levels of sex-steroid hormones, IGF-I and IGF-binding protein-3: a cross-sectional study in healthy women. Eur J Endocrinol 150(2):161-171

39. Kaaks R, Lukanova A, Kurzer M (2002) Obesity, endogenous hormones, and endometrial cancer risk: a synthetic review. Cancer Epidemiol Biomarkers Prev 11(12):1531-1543

40. Verkasalo P, Thomas H, Appleby P et al (2001) Circulating levels of sex hormones and their relation to risk factors for breast cancer: a cross-sectional study in 1092 pre- and postmenopausal women (United Kingdom). Cancer Causes Control 12 (1):47-59

41. Golden S, Dobs A, Vaidya D et al (2007) Endogenous sex hormones and glucose tolerance status in post-menopausal women. J Clin Endocrinol Metab 92(4):1289-1295 